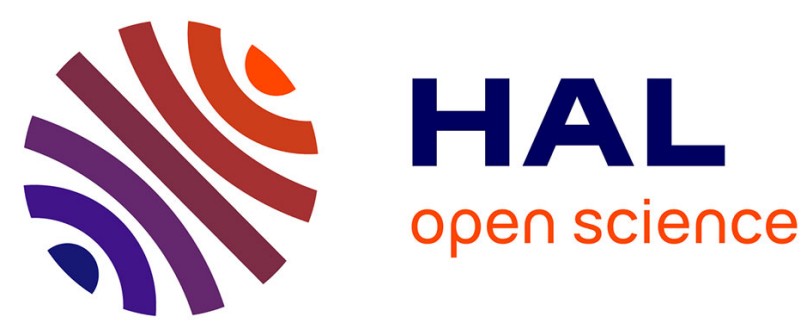

\title{
Rotation movement based on the Spatial Fourier Interpolation Method (SFIM)
}

Laurent Montier, Stéphane Clénet, Thomas Henneron, Benjamin Goursaud

\section{To cite this version:}

Laurent Montier, Stéphane Clénet, Thomas Henneron, Benjamin Goursaud. Rotation movement based on the Spatial Fourier Interpolation Method (SFIM). IEEE Transactions on Magnetics, 2017, 53, pp.1-4. 10.1109/TMAG.2017.2657008 . hal-01519739

\section{HAL Id: hal-01519739 \\ https://hal.science/hal-01519739}

Submitted on 9 May 2017

HAL is a multi-disciplinary open access archive for the deposit and dissemination of scientific research documents, whether they are published or not. The documents may come from teaching and research institutions in France or abroad, or from public or private research centers.
L'archive ouverte pluridisciplinaire HAL, est destinée au dépôt et à la diffusion de documents scientifiques de niveau recherche, publiés ou non, émanant des établissements d'enseignement et de recherche français ou étrangers, des laboratoires publics ou privés. 


\title{
Rotation movement based on the Spatial Fourier Interpolation Method (SFIM)
}

\author{
L. Montier ${ }^{1,2}$, S. Clénet ${ }^{1}$, T. Henneron ${ }^{1}$ and B. Goursaud ${ }^{2}$ \\ ${ }^{1}$ Univ. Lille, Arts et Métiers Paris Tech - L2EP - Laboratoire d'Electrotechnique et d'Electronique de Puissance, F-59000 Lille, France \\ ${ }^{2}$ EDF R\&D, THEMIS, 7 Boulevard Gaspard Monge, 91120 Palaiseau, France \\ laurent.montier@ensam.eu
}

In the field of computational electromagnetics, taking into account the rotation of a sub-domain is required to simulate certain devices such as electrical machines. Several methods have been proposed in the literature, but they remain quite difficult to implement. In this paper, we propose a sliding surface method based on a spatial Fourier interpolation in order to take into account any rotation angle with a very simple numerical implementation.

Index Terms-Finite Element Method, Fourier Series, Rotation of a Subdomain, Electrical Machine

\section{INTRODUCTION}

Modeling electrical machines with the Finite Element (FE) method is now common since it allows to obtain accurate results on complex geometries. Moreover, it is possible to model the nonlinear behaviour of ferromagnetic materials with this technique. Furthermore, the FE method model can be coupled with electric circuit equations to model the electrical environment of the machine. To take into account the motion of the rotor, several methods have been proposed in the literature. These approaches can be classified in two categories. The first one consists in computing the motion interaction inside a third thin subdomain located in the air gab between the rotor and the stator. The second one carries out the motion by ensuring a certain continuity of the potentials along a sliding interface, also located in the air gap.

In the first category, the remeshing technique is sometimes used. It consists in moving the mesh of the rotor at the desired position, and only remeshing the thin subdomain. Although it is quite simple to implement, this methods suffers from its computational cost and its varying number of unknowns at each different position. The moving band technique [1] and the Overlapping Finite Element Method [2] are more advanced approaches which allow to tackle those issues, at the cost of a more complicated implementation. Furthermore, Demenko has proposed in the moving band framework a method based on trigonometric polynomial interpolation [3].

In the sliding surface category, the Locked-Step Method (LSM) [4] might be the most used approach. It consists in permuting unknowns along the interface of a regular mesh, which leads to the exact solution for discrete values of the rotation angle. However, this approach is restricted to constant speed rotation cases and requires to remesh the interface for each different angular velocity. Thus, the Polynomial Interpolation Method (PIM) [5], the Mortar ELement Method (MEM) [6] [5] or the Lagrange Multipliers (LM) [7] have been developed to tackle these issues. However, these approaches require complex geometrical integration, matrix inversions associated with the interface of the stator and the rotor, or more advanced numerical techniques.

In this paper, the Spatial Fourier Interpolation Method (SFIM) is proposed. The SFIM allows to model any rotation by considering the motion in the Fourier domain. Besides offering very precise results, this method doesn't require any complex numerical calculations and is moreover simple to implement.

\section{Finite Element Model of AN ELECTRICAL MACHINE}

Let us consider a magnetostatic field problem of a 2D synchronous generator in a domain $D$ of boundary $\Gamma$. The machine is composed of a rotor of domain $D^{R}$ and a stator of domain $D^{S}$ where the nonlinear behaviour of their ferromagnetic material is taken into account. Furthermore, let $\Sigma$ be the common interface of $D^{R}$ and $D^{S}$ located on the airgap, as shown on figure 1. Four stranded inductors of currents $i_{k}, k=0, \ldots, 3$ are considered, $i_{0}$ being the direct current supplying the rotor inductor.

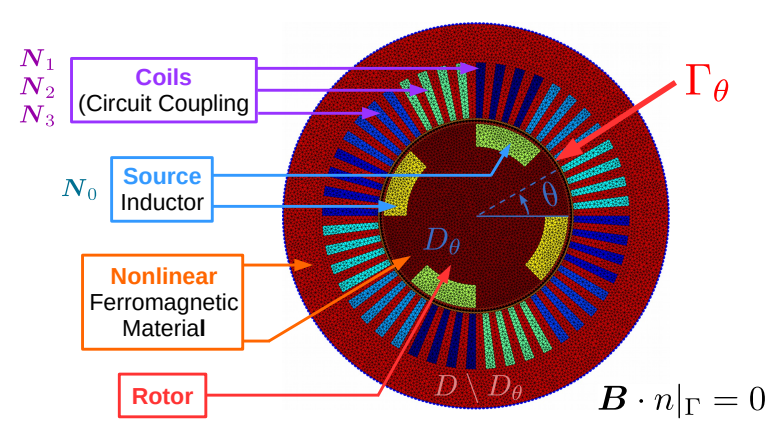

Fig. 1. Electrical machine (NOM DES DOMAINES A MODIFIER!)

\section{A. Nonlinear Magnetostatic Problem}

When the rotor is in its initial position, the Maxwell equations locally describing the magnetostatic problem are: 


$$
\begin{aligned}
\operatorname{curl} \boldsymbol{H}(\boldsymbol{x}) & =\sum_{j=0}^{3} i_{j} \boldsymbol{N}_{j}(\boldsymbol{x}), \\
\operatorname{div} \boldsymbol{B}(\boldsymbol{x}) & =0,
\end{aligned}
$$

where $\boldsymbol{H}$ denotes the magnetic field, $\boldsymbol{B}$ the magnetic flux density and $\boldsymbol{N}_{j}$ the unit current density vector flowing through the $j^{\text {th }}$ stranded inductor.

In order to solve this problem, one has to add a constitutive relation allowing to link the magnetic field $\boldsymbol{H}$ and the magnetic flux density $\boldsymbol{B}$. In the case of a nonlinear isotropic ferromagnetic material, this relation reads: $\boldsymbol{H}=\nu_{(\|\boldsymbol{B}\|)} \boldsymbol{B}$ with $\nu$ the magnetic reluctivity of the material.

Moreover, the uniqueness of the solution of this $2 \mathrm{D}$ problem is ensured by adding the following boundary condition:

$$
\boldsymbol{B} \cdot \boldsymbol{n}=0 \quad \text { on } \quad \Gamma .
$$

Finally, the vector potential $\boldsymbol{A}$ is used in order to solve this problem. It may be defined from (2) and (3) such as:

$$
\boldsymbol{B}(\boldsymbol{x})=\operatorname{curl} \boldsymbol{A}(\boldsymbol{x}) \quad \text { with } \quad \boldsymbol{A} \times \boldsymbol{n}=0 \text { on } \Gamma .
$$

By combining the constitutive relation, (1) and (4), the nonlinear problem (5) obtained is:

$$
\operatorname{curl}\left(\nu_{(\|\boldsymbol{B}\|)} \operatorname{curl} A(x)\right)=\sum_{j=0}^{3} i_{j} \boldsymbol{N}_{j}(x) .
$$

\section{B. Discretization of the problem with the Finite Element} method

By assuming that the unknown vector field $\boldsymbol{A}$ is orthogonal to the plane of study of normal $\boldsymbol{n}_{\perp}$, the problem is discretized on a 2D mesh composed of $N$ nodes, with a Dirichlet Boundary conditions on $\Gamma$. As it may be done for modeling the motion of a subdomain [8], the FE method is applied independently on the rotor part $D^{R}$ and the stator part $D^{S}$ (the SFIM will later on allow to link these two domains in order to model the motion of the rotor). To that end, the $m$ unknowns on $\Sigma$ are duplicated such that the $N^{R}$ unknowns of the rotor are dissociated from the $N^{S}$ unknowns of the stator. Thus, the two FE approximations $\boldsymbol{A}^{R}(\boldsymbol{x})=A^{R}(\boldsymbol{x}) \boldsymbol{n}_{\perp}$ and $\boldsymbol{A}^{S}(\boldsymbol{x})=A^{S}(\boldsymbol{x}) \boldsymbol{n}_{\perp}$ of the vector potential $\boldsymbol{A}(\boldsymbol{x})$ read:

$$
\begin{aligned}
& A^{R}(\boldsymbol{x})=\sum_{k=1}^{N^{R}} X_{k}^{R} w_{k}^{R}(\boldsymbol{x}), \\
& A^{S}(\boldsymbol{x})=\sum_{k=1}^{N^{S}} X_{k}^{S} w_{k}^{S}(\boldsymbol{x}),
\end{aligned}
$$

where $X_{k}^{R}$ and $X_{k}^{S}$ denote the unknown associated to the $k^{\text {th }}$ node in the rotor and the stator part respectively. Moreover $w_{k}^{R}(\boldsymbol{x})$ denotes the FE shape function associated to the $k^{t h}$ node of the rotor part, and which is always null in the stator part $D^{S}$. In the same way, its counterpart $w_{k}^{S}(\boldsymbol{x})$ is null in the rotor part $D^{R}$.
Since $\Sigma$ is located in the air gap where the magnetic reluctivity is linear, the discretization of the two independent problems (5) in $D^{R}$ and $D^{S}$ reads:

$$
\left(\begin{array}{cc}
\mathcal{M}^{R}\left(\boldsymbol{X}^{R}\right) & 0 \\
0 & \mathcal{M}^{S}\left(\boldsymbol{X}^{S}\right)
\end{array}\right)\left(\begin{array}{l}
\boldsymbol{X}^{R} \\
\boldsymbol{X}^{S}
\end{array}\right)=\left(\begin{array}{c}
\mathcal{F}_{0} i_{0} \\
\sum_{j=1}^{3} \mathcal{F}_{j} i_{j}
\end{array}\right),
$$

with $\mathcal{M}^{R}\left(\boldsymbol{X}^{R}\right)$ the stiffness matrix also accounting for the nonlinear behavior of the ferromagnetic material in the rotor, and $\mathcal{M}^{S}\left(\boldsymbol{X}^{S}\right)$, its counterpart for the stator. $\boldsymbol{X}^{R}=$ $\left(X_{k}^{R}\right)_{k=1, \ldots, N^{R}}$ denotes the unknown vector in $D^{R}$, and $\boldsymbol{X}^{S}=\left(X_{k}^{S}\right)_{k=1, \ldots, N^{S}}$, its counterpart for the stator. As for $\mathcal{F}_{k}, k=0, \ldots, 3$, they refer to the discretization of the unitary current density flowing through the $k^{\text {th }}$ stranded inductor.

However, the nonlinear problem (8) is not well defined. Indeed, boundary relations on $\Sigma$ have to be taken into account because of the domain decomposition approach used on $D^{R}$ versus $D^{S}$. If the rotor has not moved from its initial position, this relation is simply $\left.A^{R}(\boldsymbol{x})\right|_{\Sigma}=\left.A^{S}(\boldsymbol{x})\right|_{\Sigma}$. The next section is presenting how to model any position of the rotor through the SFIM.

\section{Motion OF THE ROTOR With THE SFIM}

The Spatial Fourier Interpolation Method consists in modeling the motion of the rotor subdomain $D^{R}$ versus the stator subdomain $D^{S}$, by ensuring continuity of $\boldsymbol{A}$ along the sliding interface $\Sigma$. Unlike the other sliding interface techniques, the continuity of $\boldsymbol{A}$ is ensured through a Fourier decomposition related to the curvilinear coordinates along $\Sigma$.

In order to apply further on the SFIM, the common interface $\Sigma$ separating $D^{R}$ from $D^{S}$ is composed of $m$ equispaced nodes by an angle $\Delta \theta=2 \pi / \mathrm{m}$. Moreover, we denote by $\boldsymbol{X}_{\Sigma}^{R}$, the values of $\boldsymbol{X}^{R}$ accounting for the nodes located on $\Sigma$ and $\boldsymbol{X}_{\Sigma}^{S}$, its counterpart in $D^{S}$. Let us further assume that $\boldsymbol{X}_{\Sigma}^{S}$ and $\boldsymbol{X}_{\Sigma}^{R}$ are sorted such that their $i^{t h}$ component corresponds to the node located at the angle $\theta_{i}=(i-1) \Delta \theta$, as shown on figure 2 .

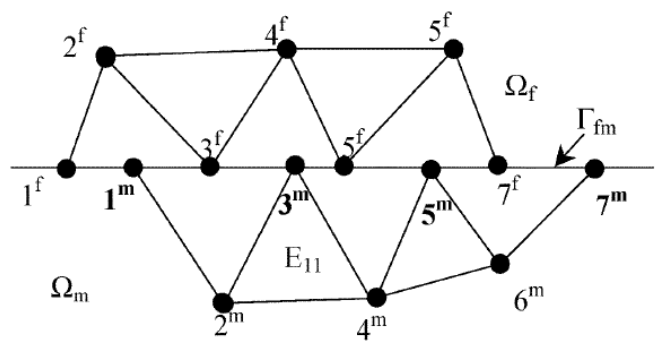

Fig. 2. Mesh along the sliding interface $\Sigma$ (A REFAIRE!!!!)

\section{A. Motion boundary relation along the sliding interface}

Let $\theta_{r}$ be the rotation angle of the rotor, with $\theta_{r}=0$ accounting for the initial position. In order to model the rotation interactions between the two independent subdomains $D^{S}$ and $D^{R}$ along the sliding interface $\Sigma$, the motion boundary relation to be ensured is in the $2 \mathrm{D}$ case:

$$
\left.A^{R}\left(r, \theta-\theta_{r}\right)\right|_{\Sigma}=\left.A^{S}(r, \theta)\right|_{\Sigma}, \forall \theta \in[0,2 \pi],
$$


where $(r, \theta)$ denotes the polar coordinates centered along the rotation axis, as shown in figure 1.

With the MEM or the LM, this relation is ensured in a weak sense [8]. This is achieved by integrating along $\Sigma$ the equation (9) multiplied with the shape functions of the nodes located on $\Sigma$. With the PIM, the motion equation (9) is approximated by using a polynomial interpolation of $\boldsymbol{X}_{\Sigma}^{S}$ with the values of $\boldsymbol{X}_{\Sigma}^{R}$. As for the LSM, the equation (9) is strictly ensured by permuting the unknowns $\boldsymbol{X}_{\Sigma}^{R}$ along $\Sigma$. However in this case, only discrete values of $\theta_{r}$, corresponding to multiples of $\Delta \theta$, can be taken into account.

The proposed approach consists in modeling the rotation of angle $\theta_{r}$ by adding a phase-lag (of amplitude $\theta_{r}$ ) between $a^{R}(\theta)$ and $a^{S}(\theta)$ in the Fourier domain, where these two functions are defined by:

$$
\begin{array}{ll}
a^{R}(\theta): & \left.\theta \rightarrow A^{R}(r, \theta)\right|_{\Sigma} \\
a^{S}(\theta): & \left.\theta \rightarrow A^{S}(r, \theta)\right|_{\Sigma} .
\end{array}
$$

Indeed, the Fourier domain has two main advantages for this kind of problem. Firstly, the two functions $a^{R}(\theta)$ and $a^{S}(\theta)$ are by definition $2 \pi$-periodic. Hence, their Fourier spectrum is a discrete set, the so-called harmonic frequencies. This means that all the information contained in $a^{R}(\theta)$ and $a^{S}(\theta)$ can be extracted onto a finite number of harmonics (under a further assumption). Secondly, adding a delay of amplitude $\theta_{r}$ to a periodic signal is performed in the Fourier domain by simply multiplying its $k^{t h}$ harmonic with $e^{j k \theta_{r}}$.

\section{B. Spatial Fourier Interpolation Method}

Let us assume that the periodic signal $a^{R}(\theta)$ does not produce harmonics of frequencies higher than the half of the sampling frequency $f_{\Delta}=1 / \Delta \theta=m /(2 \pi)$. Then, the sampling theorem implies that the Fourier Transform $\hat{a}^{R}(f)$ of $a^{R}(\theta)$ is equal to the Discrete Fourier Transform $\boldsymbol{Y}_{\Sigma}^{R}$ of $\boldsymbol{X}_{\Sigma}^{R}$ (i.e. the signal $a^{R}(\theta)$ sampled at the frequency $f_{\Delta}$ ). In other words, given that the number $m$ of nodes along $\Sigma$ is sufficiently large, the signal $a^{R}(\theta)$ can be reconstructed without loss from the DFT $\boldsymbol{Y}_{\Sigma}^{R}$ of the samples $\boldsymbol{X}_{\Sigma}^{R}=$ $\left(a^{R}\left(\theta_{i}\right)\right)_{i=1, \ldots, m}$, with $\theta_{i}=(i-1) \Delta \theta$ :

$$
\begin{aligned}
a^{R}(\theta) & =\int_{-\infty}^{\infty} \hat{a}^{R}(f) e^{-2 j \pi f \theta} \mathrm{d} f \\
& =\sum_{k=-[m / 2]}^{[(m+1) / 2]} Y_{\Sigma, k}^{R} e^{-2 j k \pi \theta},
\end{aligned}
$$

where [.] accounts for the floor function. $Y_{\Sigma, k}^{R}$ denotes the $k^{t h}$ $m$-cyclic component of the DFT $\boldsymbol{Y}_{\Sigma}^{R}$. The definition of the DFT components $\boldsymbol{Y}_{\Sigma}^{R}$ is given by:

$$
\boldsymbol{Y}_{\Sigma}^{R}=M_{f} \boldsymbol{X}_{\Sigma}^{R}
$$

with $\boldsymbol{M}_{f}=\left(m^{-1 / 2} e^{j \Delta \theta(i-1)(k-1)}\right)_{(i, k) \in[1: m]^{2}}$, the DFT matrix of size $m$. Moreover, $\boldsymbol{M}_{f}$ is a square unitary matrix which verifies $M_{f}^{t} M_{f}=I$ where $I$ is the identity matrix.
With the same assumption on $a^{S}(\theta)$, we end up with:

$$
\begin{aligned}
a^{S}(\theta) & =\sum_{k=-[m / 2]}^{[(m+1) / 2]} Y_{\Sigma, k}^{S} e^{-2 j k \pi \theta} \\
\boldsymbol{Y}_{\Sigma}^{S} & =\boldsymbol{M}_{f} \boldsymbol{X}_{\Sigma}^{S}
\end{aligned}
$$

Then, replacing (13) and (15) into the motion relation (9) leads to the following system of equations:

$$
\boldsymbol{Y}^{S}=\boldsymbol{D}\left(\theta_{r}\right) \boldsymbol{Y}^{R}
$$

where $\boldsymbol{D}\left(\theta_{r}\right)$ is a diagonal matrix of size $m$ defined by $D_{i}\left(\theta_{r}\right)=e^{j \theta_{r}(i-1)} \forall i \in\left[1, m^{+}\right]$and $D_{m-i}\left(\theta_{r}\right)=$ $e^{-j \theta_{r} i} \forall i \in\left[1, m^{-}\right]$, where $m^{+}=[(m+1) / 2]$ and $m^{-}=$ $[\mathrm{m} / 2]$. In fact, $\boldsymbol{D}\left(\theta_{r}\right)$ is the matrix allowing to add a phase lag of amplitude $\theta_{r}$ to a signal, by multiplying its $k^{t h}$ harmonic with $e^{j \theta_{r} k}$. Its peculiar form is due to the periodicity of the DFT.

Finally, using (14), (16) and (17) leads to the discrete motion relation:

$$
\boldsymbol{X}_{\Sigma}^{S}=\boldsymbol{C}\left(\theta_{r}\right) \boldsymbol{X}_{\Sigma}^{R}
$$

with $\boldsymbol{C}\left(\theta_{r}\right)=\operatorname{Re}\left(\boldsymbol{M}_{f}^{t} \boldsymbol{D}\left(\theta_{r}\right) \boldsymbol{M}_{f}\right) \in \mathbb{R}^{m \times m}$. The real part, denoted by $\mathrm{R}(\cdot)$, is not mandatory when $m$ is odd (although it might be useful from a numerical point of view). When $m$ is even however, taking the real part of $\boldsymbol{C}\left(\theta_{r}\right)$ ensures that the $(m / 2)^{t h}$ harmonic is null, according to the sampling assumption on $a^{S}(\theta)$ and $a^{R}(\theta)$.

Finally, the SFIM proposes an explicit expression for the matrix $\boldsymbol{C}\left(\theta_{r}\right)$ which makes its numerical implementation very simple. Moreover, when $\theta_{r}=k \Delta \theta, k \in \mathbb{Z}, \boldsymbol{C}\left(\theta_{r}\right)$ is reduced to a permutation matrix. In this case, the SFIM is nothing but the LSM.

\section{Coupling the motion relation with the FE system}

In order to couple the discrete motion equation (18) with the overdetermined FE system (8), an equation elimination approach as proposed in the MEM [8] is used.

First, we assume that the unknown vector of (8) is ordered such as:

$$
\left(\begin{array}{l}
\boldsymbol{X}^{R} \\
\boldsymbol{X}^{S}
\end{array}\right)=\left(\begin{array}{c}
\boldsymbol{X}_{D}^{R} \\
\boldsymbol{X}_{\Sigma}^{R} \\
\boldsymbol{X}_{D}^{S} \\
\boldsymbol{X}_{\Sigma}^{S}
\end{array}\right)
$$

with $\boldsymbol{X}_{D}^{R}$ and $\boldsymbol{X}_{D}^{S}$ the unkown related to the rotor and the stator respectively, and not on $\Sigma$. Then, the different kind of unknowns can be linked as:

$$
\left(\begin{array}{c}
\boldsymbol{X}_{D}^{R} \\
\boldsymbol{X}_{\Sigma}^{R} \\
\boldsymbol{X}_{D}^{S} \\
\boldsymbol{X}_{\Sigma}^{S}
\end{array}\right)=\left(\begin{array}{ccc}
\boldsymbol{I} & 0 & 0 \\
0 & \boldsymbol{I} & 0 \\
0 & 0 & \boldsymbol{I} \\
\boldsymbol{C}\left(\theta_{r}\right) & 0 & 0
\end{array}\right)\left(\begin{array}{c}
\boldsymbol{X}_{D}^{R} \\
\boldsymbol{X}_{\Sigma}^{R} \\
\boldsymbol{X}_{D}^{S}
\end{array}\right)
$$

which can also be noted as

$$
\boldsymbol{X}=\boldsymbol{T}\left(\theta_{r}\right) \overline{\boldsymbol{X}}
$$


By denoting the FE system (8) with $\boldsymbol{M}(\boldsymbol{X}) \boldsymbol{X}=\boldsymbol{F}$, the full system accounting for the motion of the rotor is finally:

$$
\boldsymbol{T}^{t} \boldsymbol{M}(\overline{\boldsymbol{X}}) \boldsymbol{T} \overline{\boldsymbol{X}}=\boldsymbol{T}^{t} \boldsymbol{F}
$$

This system is sparse and positive definite. It therefore can be solved with numerical methods such as the Preconditioned Conjugate Gradient.

\section{ApPLicAtion}

The SFIM is compared to the OFEM on a 2D-FEM model of a synchronous generator composed of nonlinear ferromagnetic materials. The mesh presented in figure 1 is composed of 9105 nodes, with $m=192$ nodes along the sliding surface $\Sigma$. The inductor located in the rotor is supplied with a direct current $i_{0}$ while the three phases of the stator are connected to a resistor $R=10 k \Omega$. The rotor is driven at a constant rotation speed $\Omega_{0}$, so that it requires exactly $n_{t}=1000$ time-steps to perform a full mechanical period. Since $m$ is not a multiple of $n_{t}$, the LSM is not applicable, hence the using of the OFEM and the SFIM. The setup of the electrical machine is reminded on figure 3

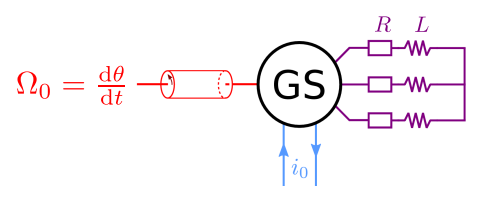

Fig. 3. Setup of the synchronous generator (à modifier (enlever L!!!))

Figures 4 shows the magnetic fluxes flowing through the three phases of the stator computed with the two methods. The curves from the SFIM matches the ones obtained with the OFEM, validating our approach.

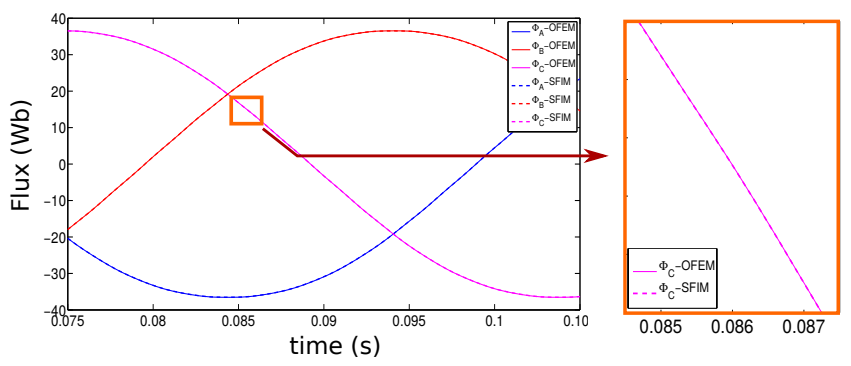

Fig. 4. magnetic fluxes through the three phases of the stator, computed with both the OFEM and the SFIM.

Figure 5 presents the electromotive forces associated with the three statoric phases. Once again, the results obtained from both models match. Moreover, the SFIM provides smoother results which makes perfect sense since it relies on a Fourier series expansion along $\theta$. Indeed in this case, the e.m.f. are related to $\frac{\mathrm{d} \boldsymbol{X}}{\mathrm{d} \theta}$.

Finally, the DFT of $a^{R}(\theta)$ computed with the OFEM is presented on figure 6 . It shows that our assumption about the limited bandwidth of $a^{R}(\theta)$ is correct.

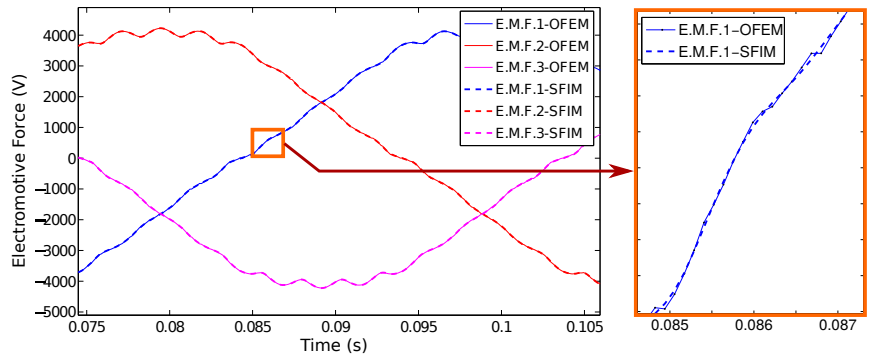

Fig. 5. Electromotive Forces computed with both the OFEM and the SFIM.

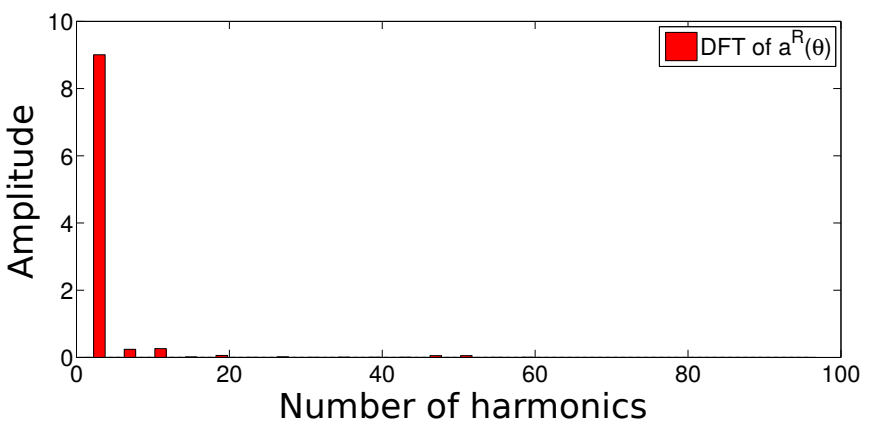

Fig. 6. Direct Fourier Transform of $a^{R}(\theta)$ computed with the OFEM

\section{CONClusion}

The Spatial Fourier Interpolation Method allows to take into account the motion of a the rotor by considering the motion equation in the Fourier domain. Although its presentation is not trivial, its implementation is straightforward because of its explicit expression. Thus, the SFIM might appear as a natural extension of the LSM through an interpolation in the Fourier space.

Future works will include its application in the 3D nodal case, and its extension to potentials discretized with edge elements.

\section{REFERENCES}

[1] B Davat, Z Ren, and M Lajoie-Mazenc. The movement in field modeling. IEEE Transactions on Magnetics, 21(6):2296-2298, 1985.

[2] Tsukerman, Igor and others. Overlapping finite elements for problems with movement. Magnetics, IEEE Transactions on, 28(5):2247-2249, 1992.

[3] Andrzej Demenko. Movement simulation in finite element analysis of electric machine dynamics. IEEE Transactions on Magnetics, 32(3):1553-1556, 1996.

[4] TW Preston, ABJ Reece, and PS Sangha. Induction motor analysis by time-stepping techniques. IEEE Transactions on Magnetics, 24(1):471-474, 1988.

[5] Xiaodong Shi, Yvonnick Le Menach, Jean-Pierre Ducreux, and Francis Piriou. Comparison between the mortar element method and the polynomial interpolation method to model movement in the finite element method. IEEE transactions on magnetics, 44(6):1314-1317, 2008.

[6] C Bernardi, Y Maday, C Mavriplis, and AT Patera. The mortar element method applied to spectral discretizations. In Proceedings of the Seventh International Conference on Finite Element Methods in Flow Problems, Huntsville, 1989.

[7] D Rodger, HC Lai, and PJ Leonard. Coupled elements for problems involving movement [switched reluctance motor]. IEEE Transactions on magnetics, 26(2):548-550, 1990.

[8] OJ Antunes, JPA Bastos, N Sadowski, A Razek, L Santandrea, F Bouillault, and F Rapetti. Comparison between nonconforming movement methods. IEEE TRANSACTIONS ON MAGNETICS MAG, 42(4):599, 2006. 